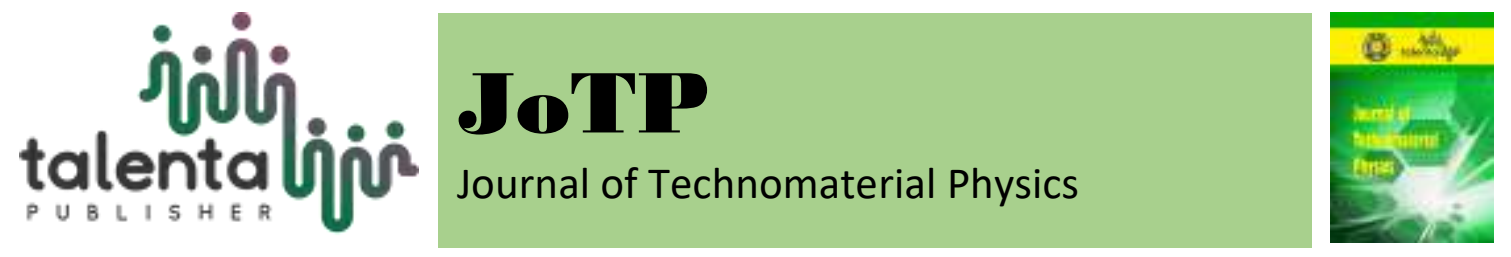

\title{
The Correlation of Blood Glucose Levels Measurement by Using Laser Speckle Imaging (LSI) Method
}

\author{
Zulkarnain ${ }^{1}$, Humaira Saufia ${ }^{2}$, Febi Oktivianda putri ${ }^{3}$ and Nur Melati ${ }^{4}$ \\ ${ }^{1,2,3,4}$ Department of Physics, Faculty of Mathematics and Natural Science, Universitas Riau 28293 \\ Indonesia
}

\begin{abstract}
Laser Speckle Imaging (LSI) method is the imaging of light interference events when it interacts on a rough surface. A laser beam which entered the surface of skin will experience reflection, refraction and scattering. Interaction between a laser beam with the surface of the skin when entering to membranes blood, provides contrast information that were resulted from the rays scattered in a certain position, recorded by a CMOS camera and translated by imageJ program. A picture will provide an information of the intensity from certain skin areas with dark light pattern which keeps blind due to the presence of blood flow that does not dwell. The result of contrast imaging was validated with direct measurement by blood glucose device. The objects were people with the age range of 17 until 63 years old. This study aims to find the correlation between contrast Speckle to blood sugar levels in the human body. The result revealed that correlation between contrast intensity of red, green and blue to glucose level are 0.3275 ; $0.3165 ; 0.3050$ respectively, and then correlation between contrast intensity to age are $0.6410 ; 0.6554 ; 0.6526$. The data indicated high correlation on contrast to age and average correlation on contrast to glucose blood level.
\end{abstract}

Keyword: Laser Speckle Imaging, Blood Glucose

Received 24 April 2019 | Revised [31 July 2019] | Accepted [31 August 2019]

\section{Introduction}

Diabetes is a disease caused by very high blood sugar (glucose) content in a person. The hormone which is responsible for controlling the amount of glucose in the blood is insulin produced by the pancreas. The factor of diabetes can be caused by two possibilities, namely because of the pancreas does not produce an adequate insulin as fuel to produce energy. Diabetes is an incurable disease, and the development of this disease is extremely rapid. Diabetes can cause serious health problems for a longer life; other effects that occur are complications of the disease from blind to disrupt the performance of the heart [1-4].

Affordable and simple methods need to be developed to find out quickly without damaging body tissues or removing blood to be tested. Currently, the blood sugar measurement tool must

\footnotetext{
*Corresponding author at: Jl. H.R. Soebrantas KM 12.5 Panam Pekanbaru 28293 Indonesia

E-mail address: zulkarnainusman@lecturer.unri.ac.id
} 
have the accuracy based on the ISO international standard. In order to be accepted, it must possess an accuracy value with an error range of $20 \%$, while the simplest tools have an accuracy of $10 \%-15 \%$.

The other sensors have been developed to monitor glucose levels. The efforts to reduce the inconvenience produced are by extracting samples in small volumes, namely on body parts such as palms, thighs and stomach. Non-invasive glucose measuring devices provide a solution to overcome this problem with optical techniques given to the skin surface [5-8]. This technique includes a near infrared spectroscopy which can investigate variations in the depth of body tissues that differ from 1 to $100 \mathrm{~mm}$ or 10 to 50 micrometers through infrared wavelength transmissions [9-12]. The non-invasive blood glucose measuring devices have been carried out by monitoring blood flow and blood oxygen levels in a few seconds [13-16]. Detect changes in blood sugar concentration in vivo can be observed by spatial diffuse reflectance [17].

The non-invasive measurement of glucose level is explained by [1] in Equation 1.

$$
C_{v}(t)=\frac{(1-\varepsilon) \cdot q_{o} \cdot h(t)}{F}
$$

Where $\mathrm{Cv}$ is the concentration of glucose in the vessels at time $\mathrm{t}$, and $\mathrm{F}$ is the blood flow (expressed in the amount of blood, usually in liters per minute). Pulse of glucose is expressed in $\mathrm{q}_{\mathrm{o}}$, this shows the amount of glucose (in $\mathrm{mg}$ ) in the blood per heart rate, whereas $\varepsilon$ is the glucose pulse fraction extracted from the blood system and metabolized, $h(t)$ is the reversible fate of glucose in the organ that causes delay and distortion that occurs in glucose pulses in vessels.

The intensity of average fluctuations at a point in a speckle pattern shows the average scattering movement rate. A very fast fluctuation can be captured by CCD cameras, by measuring the blur effect on camera lighting in certain patterns. The relationship between movement, specimen fluctuations and blur effects can easily be described as the movement of scattering produced by fluctuations in speckle patterns rather than from blur image. The degree of blur is generally calculated using a parameter called contrast, given by the symbol $\mathrm{K}$ and defined in equation 2 , namely the comparison of the standard deviation intensity to the average intensity.

$$
K \equiv \frac{\sigma}{\bar{I}}
$$

Other techniques have also been performed through Raman Spectroscopy to measure scattered light due to the effects of oscillation and rotation by glucose. Acoustic photo spectroscopy is a technique of measuring acoustic wave pressure made from heating at a certain sample area. Changes in scattering and polarization are techniques for measuring changes in scattering and polarization properties caused by glucose. Secondary interferometry pulse is a method for determining glucose concentration by measuring the refractive index group of glucose solutions using time delay in the second femto order in the time of flight method with second femto interferometry pulse [18-19]. Optical coherent tomography is a technique based on the 
measurement and analysis of interference patterns between back scattered light coherently from specific tissue layers, reference rays and several different types of fluorescence phenomena [20].

This research uses the basic operating principle of a special optical configuration to monitor glucose levels based on skin vibrations that caused by blood pulsation fluxes. This principle uses the LSI (Laser Speckle Imaging) method by arranging optical devices to observe the movement of speckle patterns generated on the part of the human skin that is targeted when emitted by a beam of laser light. The intensity speckle patterns were analyzed and compared with direct measurements of sugar levels using digital tools, which will then be observed for trends in the curve of the speckle pattern.

The research intends to design a simple tool for detecting blood sugar levels in the human body, visualizing the shape of speckles in images and graphics to determine the level of sugar. Moreover, it will compare the results of speckle intensity to medically obtained blood sugar content (blood sampling and digital measurement). The aim of this research is to model a speckle pattern on human blood sugar against medical direct measurement of the target (blood), so as to obtain a measurement system that is dynamic, comfortable and practical.

\section{Materials and Methods}

This research uses the experimental method by compiling a set of contrast measuring devices from a target. The arrangement of device is displayed in Figure 1. The research tools consist of a laser, a CCD camera, a computer, a lens, a stand and a target to be studied. The CCD camera is connected to a computer to analyze the contrast of speckles and lasers used to illuminate the target. The focus is on improving the interpretation and application of speckle contrast measurements.

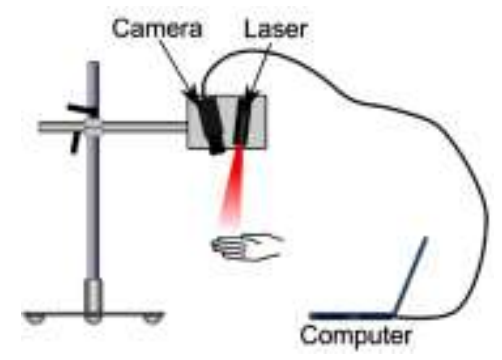

Figure 1. Laser speckle imaging set up. Object was positioned under the laser beam and captured by CCD camera. Image is then transferred into the computer for further analysis.

The research begins with determining the sample, starting from students under the age of 20 years, students aged between 20 to 25 years, lecturers aged between 30 to 40 years and lecturers aged over 40 years. Each age variation is taken with 10 times contrast test.

The measuring instrument consists of a laser, a lens, a CCD and a set of computers. Besides that, it requires equipment that has been circulating in the market in the form of Portable Easy 
Touch GCU. This tool is portable and equipped with measuring sticks to measure blood sugar levels. GCU is used as a system calibration.

The process of data retrieval was done by placing objects. In this case, the finger of the human hand used was the ring finger. This research uses a Diode laser with a wavelength of $650 \mathrm{~nm}$. The laser illuminates the object and was recorded by a CCD camera that was connected to the computer. The reflection of light that had hit the ring finger was captured by the CCD camera and translated by the ImageJ program as an image. The shape of this image was converted into contrast by calculating the gray value level. This measurement was applying a histogram image that is already available in the imageJ program. The histogram shows measured values such as minimum intensity, maximum intensity, mean and standard deviation. The Intensity value of gray value and contrast was compared to medical measurements using GCU.

The data that has been stored in the image was then converted into a graph between the speckle contrast to the sample variation (age). The contrast of the specimen to the sugar content was taken through GCU and analyzed to obtain a model or approach between the curves taken optically with the data from GCU. Through Minitab analysis, the linear regression value was revealed.

The data collection and processing techniques is shown in Figure 2. The blood sugar levels patient was first tested through Nesco Multichek, then continued with measurement of the LSI speckle pattern, then obtained on a computer with the ImageJ program.

The process of speckle image capturing is shown in Figure 2. Figure 2a shows the LSI system settings which are arranged based on certain distances and angles. Figure $2 \mathrm{~b}$ indicates the target irradiation process and Figure $2 \mathrm{c}$ shows the process of recording images in a dark box and translated by the imageJ program.

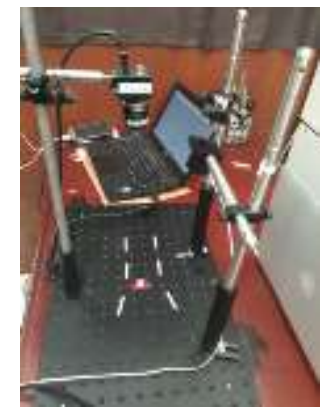

(a)

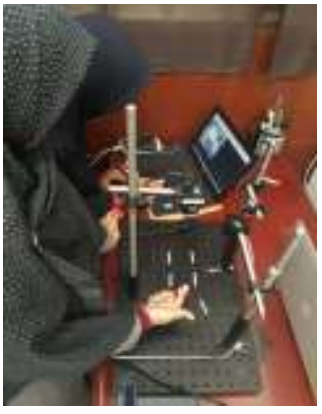

(b)

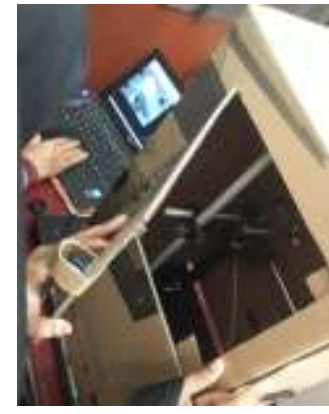

(c)

Figure 2. The scheme and technique for the imaging (a) Setting the LSI System, (b) Target is beamed with laser (c) imaging process 


\section{Result and Discussion}

The results of this research were to obtain a correlation between speckle contrast to glucose content in humans with variations in age from 17 to 63 years, and contrast of speckles to human age. The trend from contrast graphs against glucose values was measured by a correlation factor $\mathrm{R} 2$ with corresponding trend graphs namely order 5 polynomials.

The capture result on the ring finger as shown in Figure 2a, that has a form of spots was caused by the interaction of laser light against blood fluctuations, reflection and interference that form a speckle pattern or known as bio-speckle.

The speckle pattern that has been formed from by CMOS cameras used the IC Capture 2.4 program, which is the default program from the camera was then analyzed using the imageJ program. This program translates from 44200 enumerators in a few seconds. The histogram pattern is shown in Figure 3.

The histogram displays the values which are needed to be analyzed including the minimum, maximum and mean of the specimen intensity in RGB, and the standard deviation intensity value. The contrast value of RGB can be analyzed through equation 2 , which is the comparison between the standard deviation values of the average intensity.

The relationship between blood glucose levels to the mean value of intensity and contrast intensity is described in Table 1.

Table 1 The mean, intensity and contrast of blood glucos data from dari capturing and histogram.

\begin{tabular}{ccc}
\hline Blood Glucose $(\mathrm{mg} / \mathrm{dl})$ & Mean (a.u) & Contrast (a.u) \\
\hline 61 & 64.176 & 0.803822 \\
73 & 65.8061 & 0.905343 \\
75 & 52.6605 & 0.879924 \\
77 & 65.4582 & 0.84572 \\
80 & 56.6675 & 0.863736 \\
88 & 61.0178 & 0.911285 \\
96 & 59.894 & 0.820262 \\
98 & 55.4793 & 0.943049 \\
99 & 54.2559 & 0.842369 \\
99 & 74.3341 & 0.775349 \\
108 & 53.9959 & 0.803702 \\
110 & 66.3345 & 0.791175 \\
111 & 58.3929 & 0.848237 \\
132 & 65.328 & 0.743667 \\
142 & 74.4732 & 0.732955 \\
\hline
\end{tabular}


Table 1 displays the results of glucose measurements using a portable GCU tool in an invasive manner, which is taking blood using a syringe and continuing with the determination of glucose levels. The mean and contrast values were obtained from the analysis using imageJ software. In general, from the distribution of data, it can be seen that the higher the glucose level, the smaller the contrast intensity. This is caused by the relaxation of glucose content at high levels decreases when interacting with laser light. This decrease affects the reflection of light that occurs at the vibration of the skin of the hand, resulting in less diffraction and interference patterns.
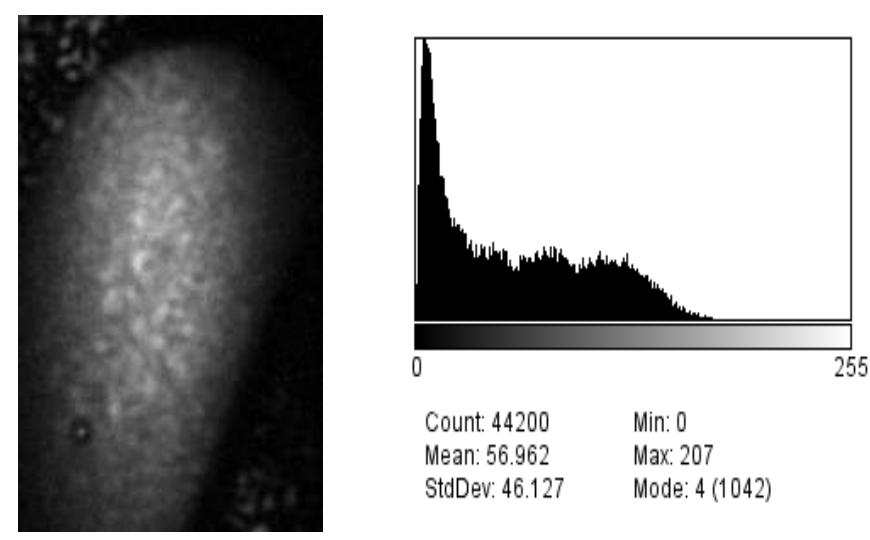

Figure 3. The speckle pattern and histogram obtained from ImageJ program

Figure 3 shows that speckle patterns of light interaction with skin roughness and blood flow to tissues under the skin. The speckle pattern that formed on the fingers was flicking, while the speckle background pattern (the base made of iron plates) was not blinking. The speckle pattern on the finger is a bio-speckle that was resulted from diffraction of a moving object, so that diffuse reflection and interference occur temporally.

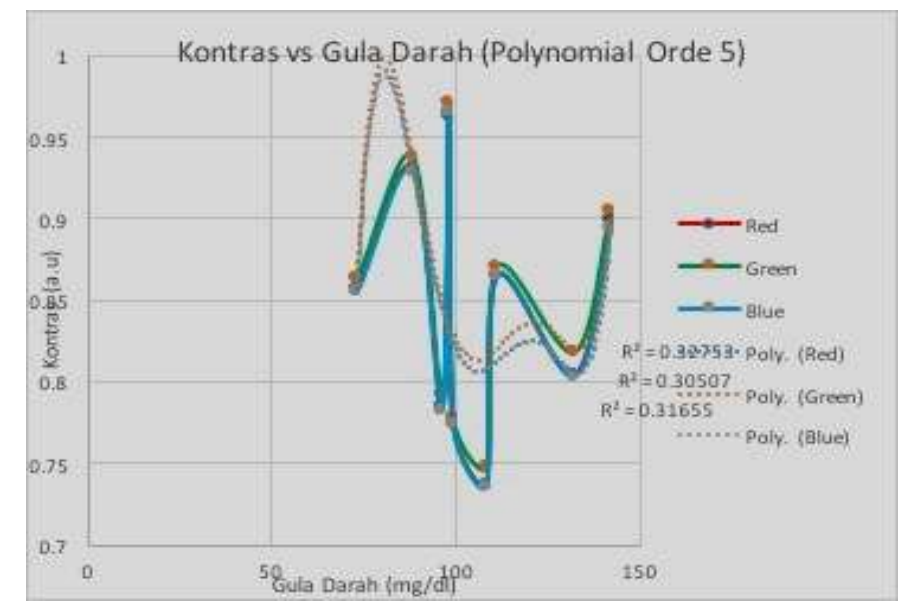

Figure 4. The graph of contrast against blood glucose level

The result of a graph plot between contrast and blood sugar is shown in Figure 4. This graph is plotted for all ages from 17 years to 63 years. It can be seen that the trend of the zigzag chart, however, has a moderate categorized correlation factor of 0.327 for red, 0.305 for green and 
0.316 for blue. This calibration of direct measurements provides an interrelated correlation between blood glucose levels and contrast intensity.

The best correlation is obtained if the factor $\mathrm{R} 2$ is 1 . The correlation value listed in the graph is depicted in order 5 polynomials to obtain an easy calibrated approach.

The graph of the relationship between contrasts to age shows a good correlation with R2 of 0.65 for red, 0.64 for green and 0.65 for blue. This correlation reveals the significant relationship between age and contrast. In other words, it can be seen that the higher the people age, the lower the contrast value. The most influencing factor is the presence of blood fluctuations which are slightly slowed down, resulting the reflection of the light that forms the diffraction pattern on the camera is getting smaller.

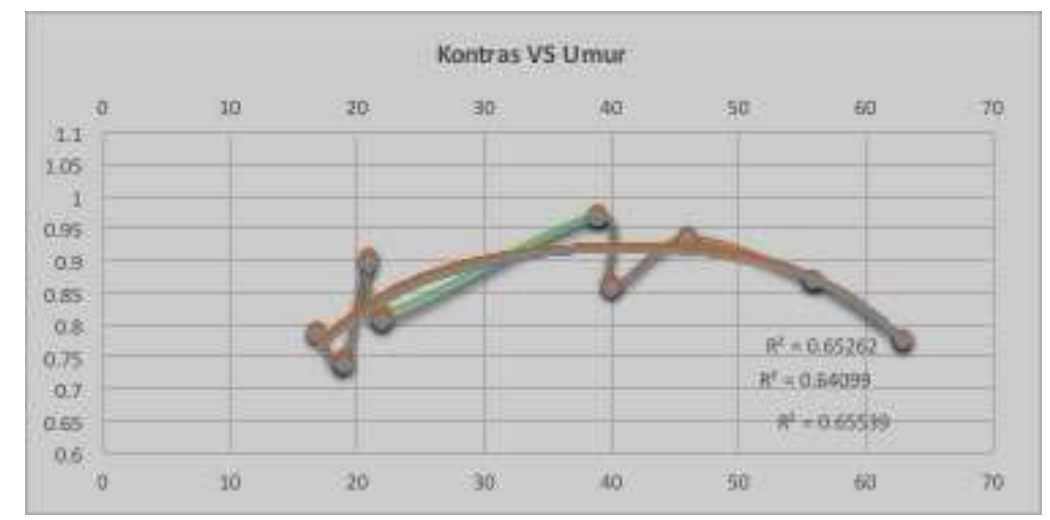

Figure 5. The graph of intensity contrast towards age

As a comparison, during the process of measuring glucose and intensity, participants were conditioned to fast and then the measurement was performed again after they ate for duration of silence of 2 hours after eating. The measurement showed an increase in blood glucose values after eating and the intensity of contrast was smaller.

\section{Conclusion}

It can be concluded that the correlation between blood sugar and intensity levels of RGB contrast had a correlation that was still considered as moderate, namely 0.3275 for Red, 0.3165 for green and 0.3050 for blue, while the correlation between contrasts to age was 0.6410 for red, 0.6554 for green and 0.6526 for blue.

\section{Acknowledgement}

Authors would like to acknowledge LPPM UNRI for funding the research via "Dosen Muda dengan DIPA tahun 2017" scheme, with the contract number of: 878/UN.19.5.1.3/PP/2017. Moreover, a gratitude is also adressed to photonic laboratorium of Universitas Riau group. 


\section{References}

[1] L. S. Jefferson, A. D. Chernington and H. M. Goodman, The Endocrine System, the Endocrine Pancreas and Regulating of Metabolism, Handbook of Physiology, Volume 2, USA: Oxford University Press, 2011.

[2] K. M. Narayan, E. W. Gregg, A. Fagot-Campagna, M. M. Engelgau and F. Vinicor, "Diabetes--A Common, Growing, Serious, Costly, and Potentially Preventable Public Health Problem," Diabetes Research and Clinical Practice, vol. 50, no. 2, pp. S77-S84, 2000.

[3] I. Wolf, S. Sadetzki, R. Catane, A. Karasik and B. Kaufman, "Diabetes Mellitus and Breast Cancer," The Lancet Oncology, vol. 6, no. 2, pp. 103-111, 2005.

[4] E. Murphy and A. L. Kinmount, "No Symptoms, No Problem? Patients' Understandings of Non-Insulin Dependent Diabetes," Family Practice, vol. 12, no. 2, pp. 184-192, 1995.

[5] R. J. McNichols and G. L. Cote, "Optical Glucose Sensing in Biological Fluids: An Overview," J. Biomed. Opt., vol. 5, no. 1, pp. 5-16, 2000.

[6] R. K. Peters and D. Elmerick, "Non-invasive Glucose Measuring Device and Method for Measuring Blood Glucose," U.S. Patent 5910109, June 8, 1999.

[7] T. A. Peyser, R. O. Potts, H. L. Berman, J. W. Moyer and M. A. Kouchnir, "Devices, Methods, and Kits for Non-Invasive Glucose Measurement," U.S. Patent 7725149, May 25, 2010.

[8] E. W. Stark, "Non-invasive Glucose Measurement Method and Apparatus," U.S. Patent 5433197, July 18, 1995.

[9] H. M. Heise, R. Marbach, G. Janatsch and J. D. Kruse-Jarres, "Multivariate Determination of Glucose in Whole Blood by Attenuated Total Reflection Infrared Spectroscopy," Anal. Chem., vol. 61, no. 18, pp. 2009-2015, 1989.

[10] J. Yadav, A. Rani, V. Singh and B. M. Murari, "Prospects and Limitations of Non-Invasive Blood Glucose Monitoring Using Near-Infrared Spectroscopy," Biomedical Signal Processing and Control, vol. 18, pp. 214-227, 2015.

[11] J. Nyström, B. Lindholm-Sethson, L. Stenberg, Stig Ollmar, J. W. Eriksson and P. Geladi, "Combined Near-Infrared Spectroscopy and Multifrequency Bio-Impedance Investigation of Skin Alterations in Diabetes Patients Based on Multivariate Analyses," Medical and Biological Engineering and Computing, vol. 41, no. 3, pp. 324-329, 2003.

[12] C. D. Brown, H. T. Davis, M. N. Ediger, C. M. Fleming, E. L. Hull and M. Rohrscheib, "Clinical Assessment of Near-Infrared Spectroscopy for Noninvasive Diabetes Screening," Diabetes Technology \& Therapeutics, vol. 7, no. 3, pp. 456-466, 2005.

[13] M. Ahmad, A. Kamboh and A. Khan, Non-invasive Blood Glucose Monitoring Using Near-infrared Spectroscopy, EDN Network, 2013.

[14] K. A. U. Menon, D. Hemachandran and T. K. Abhishek, "A Survey on Non-Invasive Blood Glucose Monitoring Using NIR," In 2013 International Conference on Communication and Signal Processing, pp. 1069-1072. IEEE, 2013.

[15] K. A. U. Menon, D. Hemachandran and A. T. Kunnath, "Voltage Intensity Based NonInvasive Blood Glucose Monitoring," In 2013 Fourth International Conference on Computing, Communications and Networking Technologies (ICCCNT), pp. 1-5. IEEE, 2013.

[16] R. R. Steuer and D. R. Miller, "Method and Apparatus for Non-Invasive Blood Constituent Monitoring," U.S. Patent 6671528, December 30, 2003.

[17] J. T. Bruulsema, M. Essenpreis, L. Heinemann, J. E. Hayward, M. Berger, F. A. Greis, T. Koschinsky, C. J. Sandhal, H. Orskov, T. J. Farell, M. S. Patterson and D. Bocker, "Detection of Changes in Blood Glucose Concentration in-vivo with Spatially Resolved Diffuse Reflectance," in Conference on Biomedical Optical Spectroscopy and Diagnostics (Optical Society of America), USA, 1996.

[18] Y. Hori, T. Yasui and T. Araki, "Multiple-Scattering-Free Optical Glucose Monitoring 
Based on Femtosecondpulse interferometry," Opt. Rev., vol. 12, no. 3, pp. 202-206, 2005.

[19] Y. Yamaoka, K. Minoshima and H. Matsumoto, "Direct Measurement of the Group Refractive Index of Air with Interferometry Between Adjacent Femtosecond Pulses," Applied Optics, vol. 41, no. 21, pp. 4318-4324, 2002.

[20] M. Kinnunen, R. Myllyla and S. Vainio, "Detecting Glucose-induced Changes in vitro and in vivo Experiments with Optical Coherence Tomography," J. Biomed. Opt., vol. 13, no. 2, pp. 021111, 2008. 